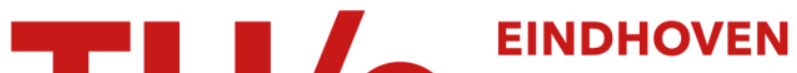

\section{Mode matching in engine ducts with vortical flows}

\section{Citation for published version (APA):}

Vilenski, G. G. (2006). Mode matching in engine ducts with vortical flows. (CASA-report; Vol. 0617). Technische Universiteit Eindhoven.

\section{Document status and date:}

Published: 01/01/2006

\section{Document Version:}

Publisher's PDF, also known as Version of Record (includes final page, issue and volume numbers)

\section{Please check the document version of this publication:}

- A submitted manuscript is the version of the article upon submission and before peer-review. There can be important differences between the submitted version and the official published version of record. People interested in the research are advised to contact the author for the final version of the publication, or visit the $\mathrm{DOI}$ to the publisher's website.

- The final author version and the galley proof are versions of the publication after peer review.

- The final published version features the final layout of the paper including the volume, issue and page numbers.

Link to publication

\section{General rights}

Copyright and moral rights for the publications made accessible in the public portal are retained by the authors and/or other copyright owners and it is a condition of accessing publications that users recognise and abide by the legal requirements associated with these rights.

- Users may download and print one copy of any publication from the public portal for the purpose of private study or research.

- You may not further distribute the material or use it for any profit-making activity or commercial gain

- You may freely distribute the URL identifying the publication in the public portal.

If the publication is distributed under the terms of Article 25fa of the Dutch Copyright Act, indicated by the "Taverne" license above, please follow below link for the End User Agreement:

www.tue.nl/taverne

Take down policy

If you believe that this document breaches copyright please contact us at:

openaccess@tue.nl

providing details and we will investigate your claim. 


\title{
Mode Matching in Engine Ducts with Vortical Flows
}

\author{
Gregory Vilenski* \\ Eindhoven University of Technology, 5600 MB Eindhoven, The Netherlands.
}

The triple-plane pressure matching method used to recover acoustic data in the region of noise generation from the CFD results is extended to the case of swirling vortical flows.

Unsteady perturbations to the mean flow are taken in the modal form. They are determined from the numerical solution of the related eigenvalue problem for the pressure mode amplitude. After the set of modal eigenfunctions has been obtained, the triple-plane matching method is used to find the approximation of the unsteady pressure field in order to determine the coefficients of the corresponding modal expansion.

The accuracy of the method is checked against the test case based on the realistic engine geometry and the CFD data supplied by Rolls-Royce and originally considered in the paper by Ovenden \& Rienstra (2004).

Practical recommendations for numerical implementation of the triple-plane matching method for vortical flows are given.

\section{Nomenclature}

$\begin{array}{ll}t, x, r, \theta & \text { time, axial, radial and circumferential coordinates } \\ u, v, w & \text { projections of the velocity vector on the coordinate axes } x, r \text { and } \theta \\ \rho, p & \text { density and pressure } \\ s & \text { hub-to-tip ratio } \\ h & \text { dimensional inner duct radius } \\ d & \text { dimensional outer duct radius } \\ \omega & \text { dimensional frequency } \\ m & \text { circumferential wavenumber } \\ k & \text { dimensional lateral wavenumber } \\ M & \text { Mach number } \\ \sim & \text { upper-case symbol denoting nondimensional values } \\ c_{0} & \text { sound speed in the flow without swirl } \\ a & \text { sound speed in swirling flow } \\ n & \text { eigenmode number }\end{array}$

\section{Introduction}

The present work extends the triple-plane pressure matching (TPPM) method originally proposed by Ovenden \& Rienstra (2004) for potential acoustic flows to the case of swirling vortical flows. The objective of the method is to extract acoustic information in the region of noise generation from the data obtained by means of direct CFD computation.

The main idea of the method is to use a set of closely spaced control surfaces in the CFD region, in order to approximate the unsteady part of the pressure by an eigenseries of the linearized Euler equations. In the potential flow case these eigenfunctions form a dense orthogonal set in an appropriate functional space,

\footnotetext{
*Research Fellow, Department of Mathematics \& Computer Science, Eindhoven University of Technology, P.O. Box 513, 5600 MB Eindhoven, The Netherlands.

Copyright (c) 2006 by G.G. Vilenski. Published by the American Institute of Aeronautics and Astronautics, Inc. with
} permission. 
and the partial sum of their Fourier series has the property of the best approximation in the relevant norm. As a result, the triple-plane pressure matching method proves to produce a very accurate and consistent approximation of the acoustic pressure field that can be subsequently used as an input information for the solution of the noise propagation problem.

In the case of a nonuniform mean flow the emergence of hydrodynamic (convected) modes and, possibly, instability waves interferes with the described procedure. Unlike the potential flow case, the structure of the spectrum is in general unknown, and the related spectral problem has to be solved numerically. The approximation properties of the set of eigensolutions are not known either.

As a result, there arisies the problem as to how the obtained eigensolutions of the linearized Euler equations should be used in order to get an accurate and computationally robust approximation of the CFD data. This is the main issue studied in the present work. The paper also extends the triple-plane matching algorithm to the case of a nonuniform mean flow and illustrates its use with the practical example originally provided in Ovenden \& Rienstra (2004). The main finding here is that the triple-plane matching method gives fairly accurate results for the case of mean flows with shear as well. If the viscous boundary-layer flow effects near the duct walls are discarded in the mean flow profile and it is approximated by an appropriate slip-stream profile, the accuracy of the approximation of the unsteady part of the pressure can be further improved in comparison with the standard TPPM method by allowing two near-wall hydrodynamic modes in the eigenseries expansion. Modelling of viscous flow effects near the duct walls results in emergence of, presumably, continuous hydrodynamic spectrum in the problem. Inclusion of such modes into the tripleplane matching procedure, in general, may make it numerically unstable and should be done with great care. Possible approach to this problem and comparison of the obtained results with the approximation based on the slip-stream mean flow profile are also discussed in the paper.

\section{Mode matching procedure}

Consider an inviscid non-heat-conducting compressible perfect gas flow inside an infinitely long straight annular duct of inner radius $h$ and outer radius $d$ as in Figure 1. Let $x, r$ and $\theta$ be the axial, the radial and the circumferential coordinates, $u, v$ and $w$ the projections of the velocity vector on the coordinate axes $x, r$ and $\theta$ respectively, $\rho$ and $p$ the density and the pressure. The dimensional equations for conservation of mass, radial, circumferential, lateral components of momentum and energy are

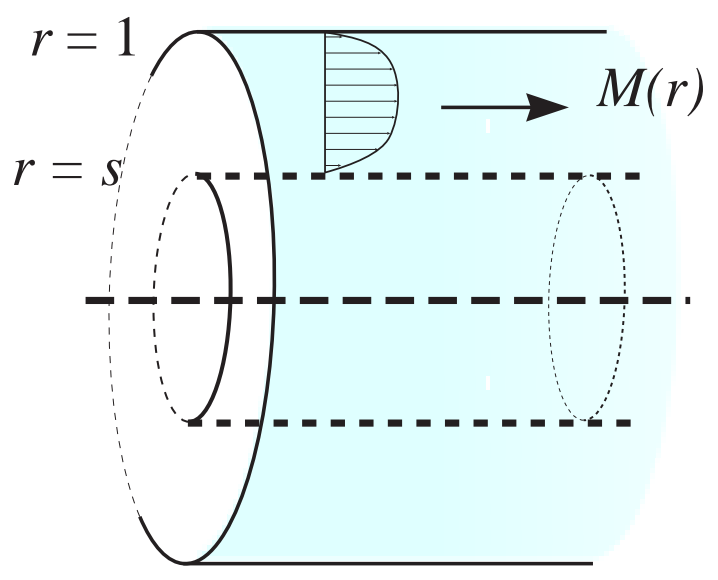

Figure 1. Flow geometry

$$
\begin{gathered}
\frac{\partial \rho}{\partial t}+\frac{1}{r} \frac{\partial(r \rho v)}{\partial r}+\frac{1}{r} \frac{\partial(\rho w)}{\partial \theta}+\frac{1}{r} \frac{\partial(\rho u)}{\partial x}=0 \\
\frac{\partial v}{\partial t}+v \frac{\partial v}{\partial r}+\frac{w}{r} \frac{\partial v}{\partial \theta}+u \frac{\partial v}{\partial x}-\frac{w^{2}}{r}=-\frac{1}{\rho} \frac{\partial p}{\partial r}, \\
\frac{\partial w}{\partial t}+v \frac{\partial w}{\partial r}+\frac{w}{r} \frac{\partial w}{\partial \theta}+u \frac{\partial w}{\partial x}+\frac{v w}{r}=-\frac{1}{r \rho} \frac{\partial p}{\partial \theta},
\end{gathered}
$$




$$
\begin{gathered}
\frac{\partial u}{\partial t}+v \frac{\partial u}{\partial r}+\frac{w}{r} \frac{\partial u}{\partial \theta}+u \frac{\partial u}{\partial x}=-\frac{1}{\rho} \frac{\partial p}{\partial x} \\
\frac{\partial p}{\partial t}+v \frac{\partial p}{\partial r}+\frac{w}{r} \frac{\partial p}{\partial \theta}+u \frac{\partial p}{\partial x}+\gamma p\left(\frac{1}{r} \frac{\partial(r v)}{\partial r}+\frac{1}{r} \frac{\partial w}{\partial \theta}+\frac{\partial u}{\partial x}\right)=0
\end{gathered}
$$

Here $t$ is time, $\gamma=c_{p} / c_{v}$ is the ratio of specific heat capacities at constant pressure and constant volume, respectively. The pressure, the density and the absolute temperature $T$ satisfy the equation of state $p=R \rho T$, $R=c_{p}-c_{v}$.

Assume that the total flow field can be decomposed into the sum of a mean base flow and small-amplitude unsteady perturbations

$$
(u, v, w, \rho, p)=(\bar{u}, \bar{v}, \bar{w}, \bar{\rho}, \bar{p})+(\widetilde{u}, \widetilde{v}, \widetilde{w}, \widetilde{\rho}, \widetilde{p}) .
$$

with the mean flow given by the formulae (see Tam \& Auriault (1998) for details)

$$
\bar{u}=\bar{u}(r), \bar{v}=0, \bar{w}=\bar{w}(r), \bar{\rho}=\bar{\rho}(r), \bar{p}=p_{d}-\int_{r}^{d} \bar{\rho}(\xi) \frac{\bar{w}^{2}(\xi)}{\xi} d \xi
$$

In the context of the triple plane matching approach (see Ovenden \& Rienstra (2004)), which objective is to extract acoustic information in the region of noise generation from the CFD solution, the mean flow (7) can be in practice obtained by means of circumferential and time averaging of the CFD data.

The variable part of the flow can be Fourier decomposed in the excitation frequency $\widetilde{\omega}$ and circumferential order $m$ :

$$
(\widetilde{u}, \widetilde{v}, \widetilde{w}, \widetilde{\rho}, \widetilde{p})(x, r, \theta, t)=\sum_{n} \sum_{m}\left(u_{n m}, v_{n m}, w_{n m}, \rho_{n m}, p_{n m}\right) \exp (-i \widetilde{\omega} n t+i m \theta) .
$$

The components $\left(u_{n m}, v_{n m}, w_{n m}, \rho_{n m}, p_{n m}\right)$ are the known functions of the radial coordinate $r$ for a given set of closely spaced control surfaces $x=x_{l}, l=0,1,2, \ldots$ in the CFD region. Normally, they can be provided by the Fourier transform of the CFD data

$$
\left(u_{n m}, v_{n m}, w_{n m}, \rho_{n m}, p_{n m}\right)=\frac{\widetilde{\omega}}{4 \pi^{2}} \int_{0}^{2 \pi / \widetilde{\omega}} \int_{0}^{2 \pi}(\widetilde{u}, \widetilde{v}, \widetilde{w}, \widetilde{\rho}, \widetilde{p}) \exp (i \widetilde{\omega} n t-i m \theta) d \theta d t .
$$

In order to obtain the representation of the unsteady part of the pressure field consistent with the Euler equations linearized on the mean flow (7), we approximate the perturbation pressure $p_{n m}(r, x)$ by the series expansion based on the eigenfunctions of the related linearized Euler equations, i.e.,

$$
p_{n m}(r, x)=\sum_{(\mu)} A_{\mu} P_{\mu}(r) \exp \left(i k_{\mu} x\right) .
$$

Here the complex numbers $A_{\mu}$ are the unknown modal amplitudes, $P_{\mu}(r)$ are the modal eigenfunctions and $k_{\mu}$ are the corresponding lateral wave numbers. $P_{\mu}(r)$ and $k_{\mu}$ satisfy the modal equation (11)

$$
P_{\mu}^{\prime \prime}+\left(\frac{\lambda+B r}{\lambda r}+C+\frac{\bar{\rho} A}{\lambda} \frac{d}{d r}\left(\frac{\lambda}{\bar{\rho} A}\right)\right) P_{\mu}^{\prime}+\left(\frac{A \Omega}{\lambda^{2}}+\frac{\bar{\rho} A}{\lambda r} \frac{d}{d r}\left(\frac{B r}{\bar{\rho} A}\right)+\frac{B C}{\lambda}\right) P_{\mu}=0,
$$

and the slip-stream boundary conditions on the inner and outer wall of the duct, respectively,

$$
P_{\mu}^{\prime}+\frac{B}{\lambda} P_{\mu}=0 \text { on } r=h, d
$$

Here primes denote differentiation with respect to $r, \omega=\widetilde{\omega} n$,

$$
\begin{gathered}
\lambda=-\omega+k \bar{u}+m \bar{w} / r, \\
A=\lambda^{2}+\frac{\bar{w}^{4}}{\bar{a}^{2} r^{2}}-\frac{\bar{w}^{2}}{r} \frac{\bar{\rho}^{\prime}}{\bar{\rho}}-\frac{2 \bar{w}}{r}\left(\bar{w}^{\prime}+\frac{\bar{w}}{r}\right), B=\frac{2 m \bar{w}}{r^{2}}-\frac{\lambda \bar{w}^{2}}{\bar{a}^{2} r}, \\
C=\frac{\bar{w}^{2}}{\bar{a}^{2} r}-\frac{1}{\lambda}\left(\frac{m}{r}\left(\bar{w}^{\prime}+\frac{\bar{w}}{r}\right)+k \bar{u}^{\prime}\right), \Omega=\frac{\lambda^{2}}{\bar{a}^{2}}-k^{2}-\frac{m^{2}}{r^{2}}, \bar{a}^{2}=\frac{\gamma \bar{p}}{\bar{\rho}} .
\end{gathered}
$$


For zero mean circumferential velocity $\bar{w}=0$ and constant mean flow density $\bar{\rho}$ the problem $(11,12)$ reduces to the Pridmore-Brown equation (13)

$$
P_{\mu}^{\prime \prime}+\beta(r) P_{\mu}^{\prime}+\gamma(r) P_{\mu}=0
$$

where

$$
\beta(r)=\left(\frac{1}{r}+\frac{2 k \bar{u}^{\prime}}{\omega-k \bar{u}}\right), \gamma(r)=\left(\frac{(\omega-k \bar{u})^{2}}{c_{0}^{2}}-k^{2}-\frac{m^{2}}{r^{2}}\right) \text { and } c_{0}^{2}=\frac{\gamma p_{d}}{\bar{\rho}} .
$$

with the boundary conditions

$$
P_{\mu}^{\prime}=0 \text { on } r=h, d .
$$

As is well known (see for instance, Eversman (1991), Tam \& Auriault (1998), Golubev \& Atassi (1998), Nijboer (2001), Cooper \& Peake (2005)), the spectrum of the problem (11,12), and similarly $(13,14)$, consists of the acoustic modes which propagate with the speed of sound and hydrodynamic modes which are convected with the local flow speed. At least theoretically, in the expansion (10) summation over both acoustic and hydrodynamic modes must be considered. For convenience of notation we divide all modes into the following three subsets: $\mu_{-}=\left\{\mu: \operatorname{Im}\left(k_{\mu}\right)<0\right\}$ - the modes with negative imaginary part of the axial wave number, $\mu_{0}=\left\{\mu: \operatorname{Im}\left(k_{\mu}\right)=0\right\}$ - the modes with zero imaginary part of the axial wave number and $\mu_{+}=\left\{\mu: \operatorname{Im}\left(k_{\mu}\right)>0\right\}$ - the modes with positive imaginary part of the axial wave number. Were the spectrum purely acoustic, say, in the absence of mean flow, the sets $\mu_{-}$and $\mu_{+}$would correspond to the decaying left and right running modes, respectively, while $\mu_{0}$ would consist of propagating modes. In the more general case of equation (11), owing to the appearance of hydrodynamic spectrum and possibly convective flow instabilities, the sets $\mu_{-}, \mu_{+}$and $\mu_{0}$ may also contain hydrodynamic modes.

Following the triple-plane matching method of Ovenden \& Rienstra (2004), we collocate the equation (10) at the three closely spaced control surfaces $x=x_{l}, l=0,1,2$ :

$$
\begin{aligned}
& \mathcal{P}_{0}(r)=\sum_{\mu \in \mu_{0} \cup \mu_{+} \cup \mu_{-}} A_{\mu} P_{\mu}(r) \exp \left(i k_{\mu} x_{0}\right), \\
& \mathcal{P}_{1}(r)=\sum_{\mu \in \mu_{0} \cup \mu_{+} \cup \mu_{-}} A_{\mu} P_{\mu}(r) \exp \left(i k_{\mu} x_{1}\right), \\
& \mathcal{P}_{2}(r)=\sum_{\mu \in \mu_{0} \cup \mu_{+} \cup \mu_{-}} A_{\mu} P_{\mu}(r) \exp \left(i k_{\mu} x_{2}\right) .
\end{aligned}
$$

Here $\mathcal{P}_{l}(r)=p_{n m}\left(r, x_{l}\right), l=0,1,2$, and the sums are taken over all modes. In order to proceed numerically, the infinite sums appearing on the right-hand sides of the above equations must be approximated by appropriate partial sums. This is not a straightforward procedure if part of the hydrodynamic spectrum is continuous or even countable. The reason for this is that now it cannot be guaranteed that for a given smooth function $p_{n m}(r, x)$ which satisfies boundary conditions $(14$,$) the Fourier expansion (10) in the eigen-$ functions of the problem (11-14) is convergent. Neither is it known that any two eigenfunctions associated to different eigenvalues are orthogonal to each other with any weight function. These circumstances make the choice of hydrodynamic eigenfunctions to be used in the construction of the numerical approximation based of the eigenseries (10) a difficult problem. Thus, in what follows we proceed guided by the analogy with the potential flow case, where hydrodynamic modes are absent, and then gradually broaden the mathematical model to the case of a finite number of hydrodynamic modes, in order to study their influence on the quality of the approximation. If the number of hydrodynamic modes is infinite, we can still try to account for their influence by taking a sufficiently large number of such modes. However, as it will be clear from what follows, much caution is needed in the case when part of the problem's spectrum is continuous.

Let $M_{0}$ denote the total number of propagating acoustic modes plus, possibly, the hydrodynamic modes from the set $\mu_{0}$. Similarly, let $M_{+}$and $M_{-}$denote the number of modes from the sets $\mu_{+}$and $\mu_{-}$, respectively, whose lateral wave numbers $k_{\mu}$ belong to the disk in the complex plane $k_{\mu}$ of sufficiently large radius $R$. If the number of hydrodynamic modes is finite, all of them are assumed to be taken into account either by making $R$ sufficiently large or directly in $M_{0}$, if their $\operatorname{Im}\left(k_{\mu}\right)=0$. Substituting the infinite sums in the 
system (15) by the corresponding partial sums we get the following set of equations:

$$
\begin{aligned}
& \mathcal{P}_{0}(r)=\sum_{\substack{j=0 \\
M_{0}+M_{+}-1}}^{M_{0}+M_{+}-1} B_{j} P_{j}(r)+\sum_{j=M_{0}+M_{+}}^{M_{0}+M_{+}+M_{-}-1} B_{j} P_{j}(r) e^{-i k_{j}\left(x_{2}-x_{0}\right)}, \\
& \mathcal{P}_{1}(r)=\sum_{j=M_{0}} B_{j} P_{j}(r) e^{i k_{j}\left(x_{1}-x_{0}\right)}+\sum_{j=M_{0}+M_{+}} B_{j} P_{j}(r) e^{-i k_{j}\left(x_{2}-x_{1}\right)} \text {, } \\
& \mathcal{P}_{2}(r)=\sum_{j=M_{0}}^{M_{0}+M_{+}-1} B_{j} P_{j}(r) e^{i k_{j}\left(x_{2}-x_{0}\right)}+\sum_{j=M_{0}+M_{+}}^{M_{0}+M_{+}+M_{-}-1} B_{j} P_{j}(r) .
\end{aligned}
$$

Here the modes corresponding to $j=0,1,2,3, \ldots, M_{0}-1$ belong to the set $\mu_{0}$, the modes corresponding to $j=$ $M_{0}, \ldots, M_{0}+M_{+}-1$ belong to the set $\mu_{+}$and the modes corresponding to $j=M_{0}+M_{+}, \ldots, M_{0}+M_{+}+M_{-}-1$ belong to the set $\mu_{-}$. A new set of independent variables $B_{j}$ was introduced in (16) instead of $A_{j}$ according to the following formula

$$
B_{j}=A_{j}\left\{\begin{array}{l}
e^{i k_{j} x_{0}}, \text { if } j=0,1, \ldots, M_{0}+M_{+}-1 \\
e^{i k_{j} x_{2}}, \text { if } j=M_{0}+M_{+}, \ldots, M_{0}+M_{+}+M_{-}-1 .
\end{array}\right.
$$

System (16) is overdetermined and cannot be solved exactly for $B_{j}$. Instead, the unknown coefficients $B_{j}$ can be found by means of quadratic error minimisation for the system (16) as proposed in Ovenden \& Rienstra (2004). Rescaling of the unknown Fourier coefficients according to formula (17) proposed by the above authors makes the system (16) numerically advantageous over the corresponding original system written in terms of $A_{j}$. It insures that none of the sums in the set of equations (16) contains pathologically large exponential terms which result in numerical instabilities associated with evanescent acoustic modes in the original system for $A_{j}$. If we now let

$$
\begin{gathered}
\xi_{j}(r)=P_{j}(r)\left\{\begin{array}{l}
1, \text { if } 0 \leq j<M_{0}+M_{+} \\
e^{-i k_{j}\left(x_{2}-x_{0}\right)}, \text { if } M_{0}+M_{+} \leq j<M_{0}+M_{+}+M_{-},
\end{array}\right. \\
\varsigma_{j}(r)=P_{j}(r)\left\{\begin{array}{l}
e^{i k_{j}\left(x_{1}-x_{0}\right)}, \text { if } 0 \leq j<M_{0}+M_{+} \\
e^{-i k_{j}\left(x_{2}-x_{1}\right)}, \text { if } M_{0}+M_{+} \leq j<M_{0}+M_{+}+M_{-},
\end{array}\right. \\
\chi_{j}(r)=P_{j}(r)\left\{\begin{array}{l}
e^{i k_{j}\left(x_{2}-x_{0}\right)}, \text { if } 0 \leq j<M_{0}+M_{+} \\
1, \text { if } M_{0}+M_{+} \leq j<M_{0}+M_{+}+M_{-},
\end{array}\right.
\end{gathered}
$$

multiply the first equation of the system (16) by $\overline{\xi_{l}}(r) r$, the second equation by $\overline{\varsigma_{l}}(r) r$ and the third one by $\overline{\chi_{l}}(r) r$, where the bar denotes complex conjugation, and integrate the resulting equations from $r=h$ to $r=d$, we get the following system for the unknown coefficients $B_{j}$ :

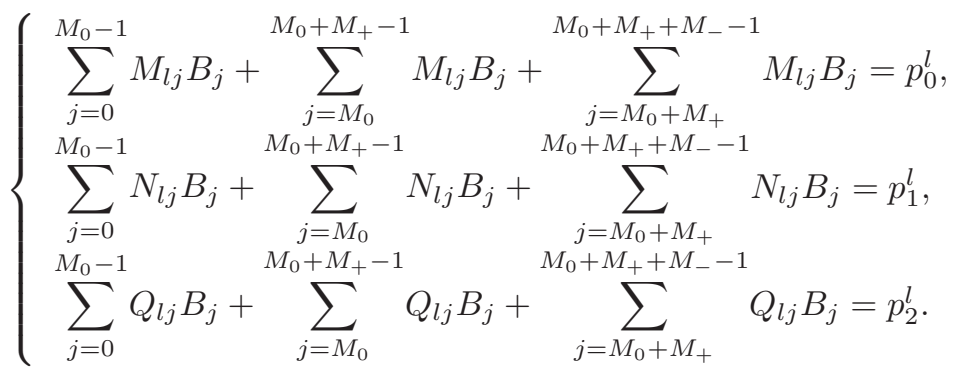

Here $l, j=0,1,2,3 \ldots M_{0}+M_{+}+M_{-}-1$,

$$
\begin{gathered}
M_{l j}=\int_{h}^{d} \xi_{j} \overline{\xi_{l}}(r) r d r, N_{l j}=\int_{h}^{d} \varsigma_{j} \overline{\varsigma_{l}}(r) r d r, Q_{l j}=\int_{h}^{d} \chi_{j} \overline{\chi_{l}}(r) r d r \\
p_{0}^{l}=\int_{h}^{d} \mathcal{P}_{0}(r) \overline{\xi_{l}}(r) r d r, p_{1}^{l}=\int_{h}^{d} \mathcal{P}_{1}(r) \overline{\varsigma_{l}}(r) r d r, p_{2}^{l}=\int_{h}^{d} \mathcal{P}_{2}(r) \overline{\chi_{l}}(r) r d r .
\end{gathered}
$$


Next, we apply the least-squares algorithm in order to minimize the objective function

$$
\left\|M B-p_{0}\right\|^{2}+\left\|N B-p_{1}\right\|^{2}+\left\|Q B-p_{2}\right\|^{2},
$$

where $B=\left(B_{0}, B_{1}, \ldots, B_{M_{0}+M_{+}+M_{-}-1}\right)$ is the vector of unknown coefficients. Matrices $M, N, Q$ and vectors $p_{0}, p_{1}, p_{2}$ are given by the relationships (19) and (20). In view of the Hermitian structure of the matrices $M, N$ and $Q$, this leads to the following algebraic system (see Ovenden \& Rienstra (2004) for details)

$$
\left(M^{2}+N^{2}+Q^{2}\right) B=M p_{0}+N p_{1}+Q p_{2}
$$

which is solved numerically for $B$. The coefficients $A_{j}$ are then recovered from formula (17).

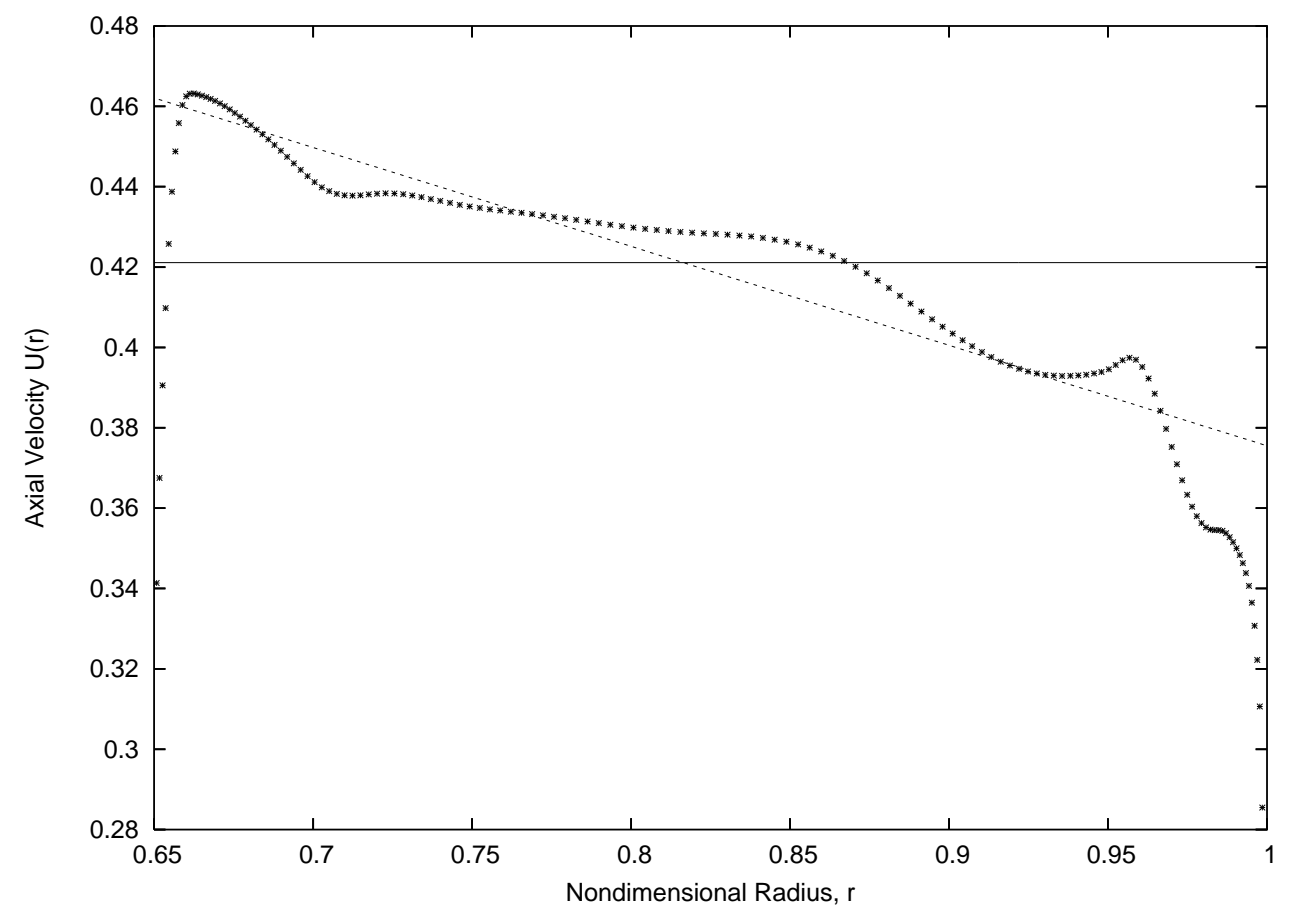

Figure 2. Mean flow axial velocity profile $M(r)=\bar{u}(r) / c(1)$ normalised by the speed of sound on the outer wall $c(1)$ versus the radial coordinate non-dimensionalized by the radius of the outer wall: asterisk - original CFD data, solid line - constant mean flow approximation, dashed line - linear approximation.

\section{Test case results for nonuniform mean flow without swirl}

In this section we check the accuracy of the method against the test case considered in detail by Ovenden \& Rienstra (2004) within the framework of potential flow theory. It is based on a realistic engine configuration and CFD data supplied by Rolls-Royce (RR) for the region downstream of the bypass outlet guide vanes, where the duct walls are almost parallel, $h=2.7 \mathrm{~m}$ and $d=4.15 \mathrm{~m}$. Detailed description of the overall engine geometry can be found in the cited paper and is omitted here. The Fourier transform of the unsteady CFD data and the circumferentially averaged mean flow is available for 11 equally spaced axial planes between $x=0.32 \mathrm{~m}$ and $x=0.36 \mathrm{~m}$. Of these we use 3 axial positions $x=0.32,0.34$ and $0.36 \mathrm{~m}$. Unsteady perturbation data corresponds to the Helmholtz number equal to 40.9, circumferential wavenumber $m=-13$, hub-to-tip ratio equal to 0.65 , and the mean flow Mach number equal to 0.43.

We consider three test cases shown in figure 2. They correspond to the approximation of the axial mean flow velocity by a uniform profile used in Ovenden \& Rienstra (2004), linear approximation of the axial mean flow profile, and the profile which mimics the original Rolls-Royce velocity distribution including viscous boundary layer behaviour near the inner and outer wall. For brevity, the latter approximation is called boundary-layer profile in what follows. Careful analysis of the original mean flow profile reveals the existence of at least three inflection points, where $\bar{u}^{\prime \prime}(r)=0$. One of these points lies near the inner wall at 
$r \doteq 0.694$, with $\bar{u} \doteq 0.446$. The other two inflection points are located near the outer wall at $r \doteq 0.97$ and $r \doteq 0.984$, the corresponded mean flow velocities being $\bar{u} \doteq 0.37$ and $\bar{u} \doteq 0.354$, respectively.

The analysis of the problem based on the generic model $(11,14)$ which allows for radial non-uniformities in density and azimuthal velocity distributions and the analysis based on Pridmore-Brown equation (13) which allows only for axial flow non-uniformity shows good agreement between the corresponding eigensolutions. Final approximations of the unsteady pressure component resulting from these two models are also close to each other. For this reason the influence of the mean flow swirl and density non-uniformity is believed to be negligible for this engine and is discarded in what follows.

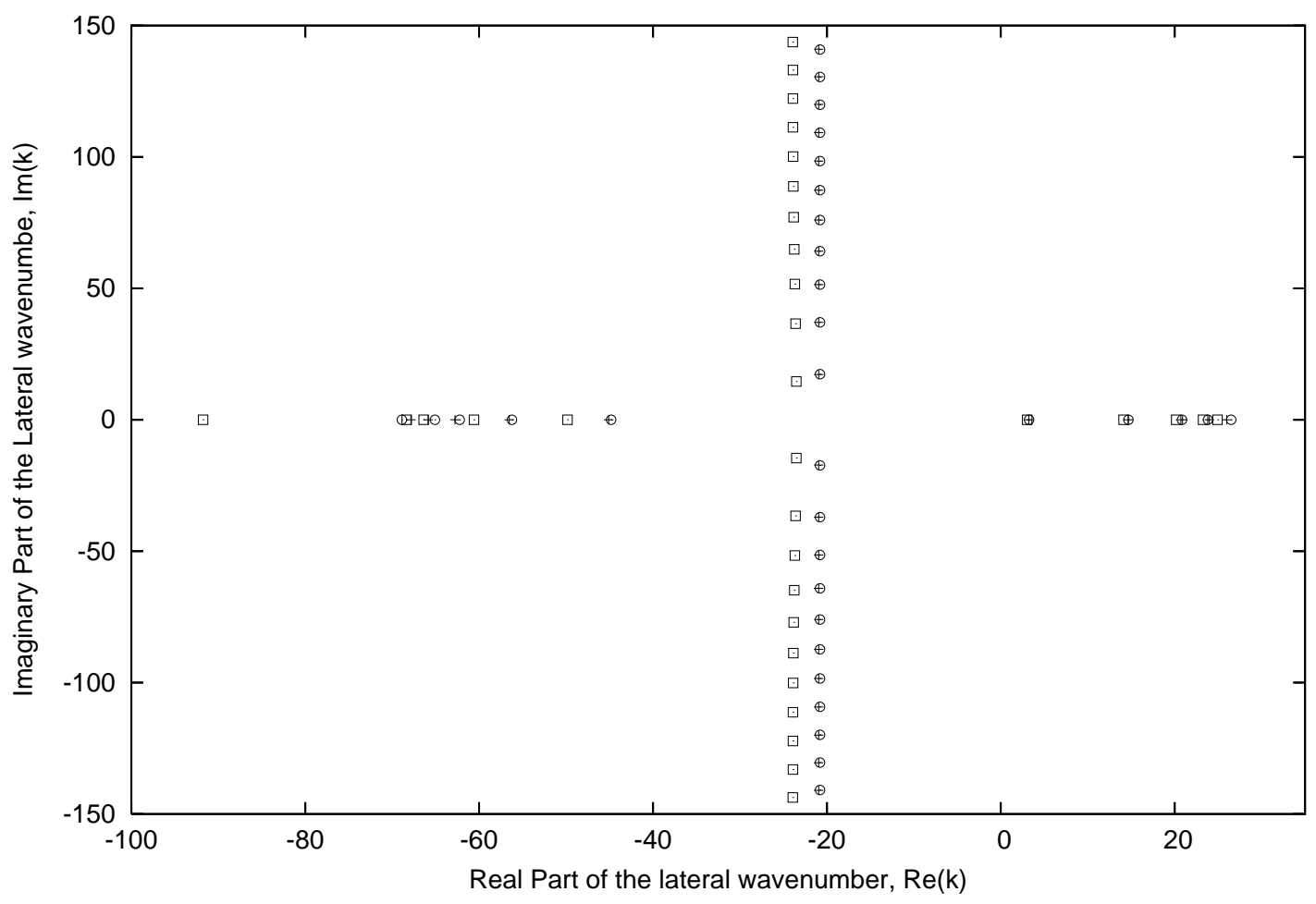

Figure 3. Acoustic part of the spectrum: + - constant mean flow profile,$\odot-$ linear mean flow profile $\square-$ mean flow profile with boundary-layer effects near the inner and the outer wall.

The patterns of the spectrum corresponding to the three approximations of the mean flow shown in figure 2 are presented in figures 3 and 4 . They show the distribution of eigenvalues in the complex plane of the spectral parameter $k_{\mu}$. The acoustic part of the spectrum for all these mean flow approximations is depicted in figure 3. It can be seen from this figure that the eigenvalues corresponding to different mean flow approximations are located fairly close to each other, suggesting that refraction of acoustic waves due to the mean flow non-uniformity is relatively unimportant for the present engine.

However, provision for mean flow non-uniformity in the model results in the appearance of hydrodynamic spectrum shown in figure 4 . It consists of only two smooth convected modes for the linear mean flow profile with $k_{h} \doteq 88.6$ and $k_{d} \doteq 108.9$. The first of these modes is localised near the hub, the second - near the outer wall. Apart from these smooth modes, it is possible to construct a continuous spectrum of hydrodynamic modes with a discontinuity in the third derivative of the pressure amplitude. These modes are not shown in the figure.

For the boundary-layer profile hydrodynamic modes are also believed to form a continuous spectrum. Several such modes obtained for 5000 grid points used to discretize Pridmore-Brown equation are shown in figure 4. Their axial wave numbers satisfy the equation $k_{i}=\omega / \bar{u}\left(r_{i}\right)$, where $r_{i}$ are the points of the computational mesh which are located in the boundary-layer region near the inner wall $r=h$ and the outer wall $r=d$. For the present mean flow profile the boundary layer thickness near each wall is estimated to be about 16 per cent of the channel width. If the number of mesh points is increased, so does the number of these hydrodynamic modes. Unfortunately, their smoothness was difficult to check numerically. 


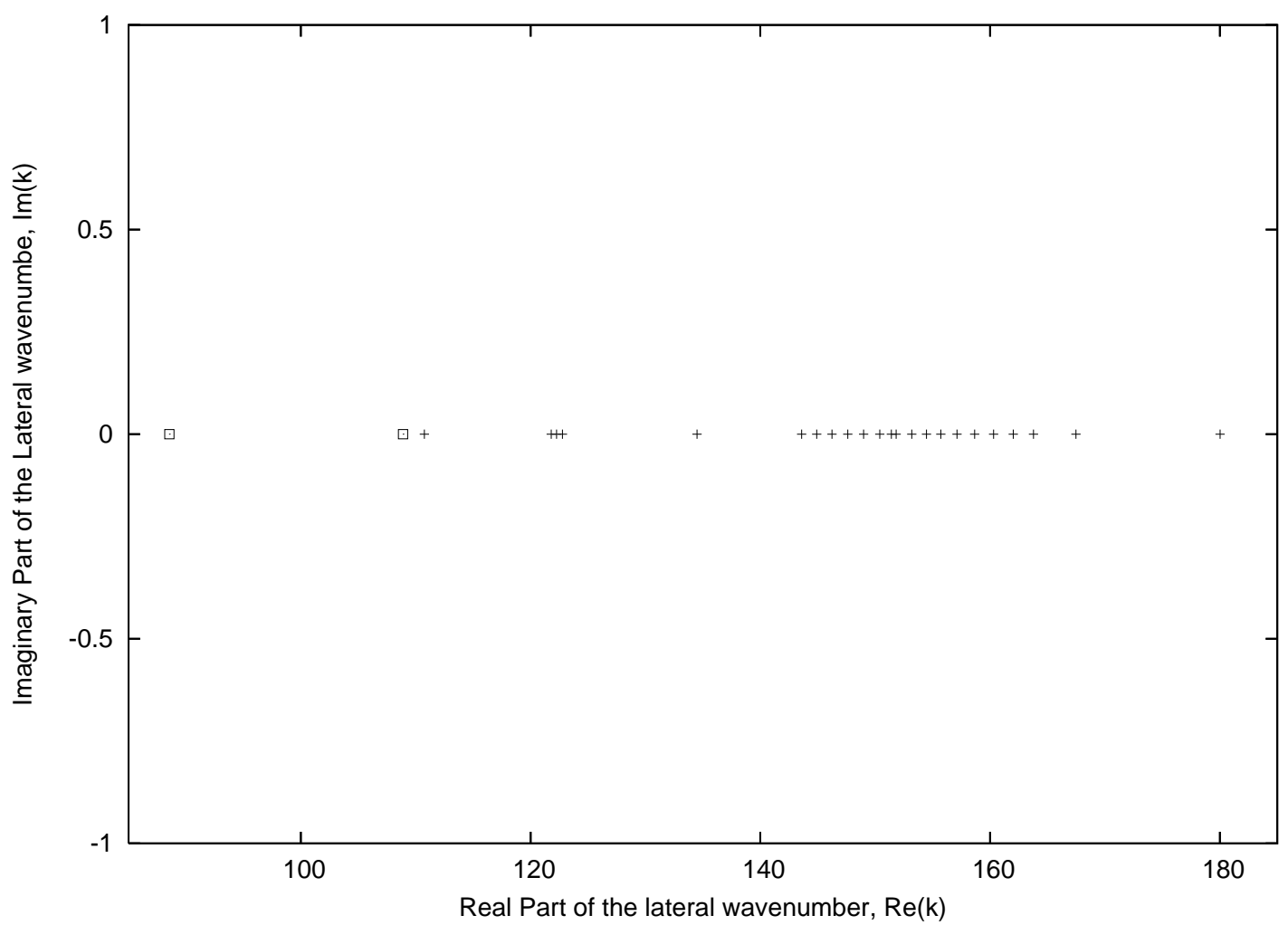

Figure 4. Hydrodynamic part of the spectrum: + - mean flow profile with boundary-layer effects near the inner and the outer wall, $\square$ - linear mean flow profile

The mode with $k_{\mu} \doteq 110$ is generated by the inflection point at $r \doteq 0.97$, and its phase speed is equal to the local mean flow velocity $\bar{u} \doteq 0.37$. Interestingly enough, this mode would be always found by the numerical code, if the number of mesh points exceeded a certain threshold limit, as opposed to the inflection point $r \doteq 0.694$, where no smooth eigenmodes were found. The three modes clustering near the point $k_{\mu} \doteq 122$ are generated by the mesh points which are localised in the vicinity of the point $r \doteq 0.984$ where both $\bar{u}^{\prime}$ and $\bar{u}^{\prime \prime}$ are close to zero. This clustering is due to the fact that we use the uniform grid with respect to $r$ in our computation. As a result, $k_{i}=\omega / \bar{u}\left(r_{i}\right)$ lie close to each other, where $\bar{u}^{\prime}(r)$ is small, and far apart, where it is large (hence the gap in the wave number range between $k_{\mu} \doteq 122, k_{\mu} \doteq 134$ and $k_{\mu} \doteq 144$ ). The wave number $k_{\mu} \doteq 151.4$ is the double eigenvalue. It corresponds to one eigenfunction located near the hub and the other one near the outer wall.

It should be noted that the clustering hydrodynamic modes, especially in the regions of low mean flow velocity gradient $\bar{u}^{\prime}(r)$, have very similar shapes and are almost linearity dependent. This is demonstrated in figure 5, where hydrodynamic modes are depicted. Owing to a very high mean flow velocity gradient near the inner wall, the mode on the left is the only mode found in the boundary-layer region near the hub. To obtain more modes in this region, the number of mesh points must be increased further. The negative-valued mode shown in a thick line corresponds to the inflection point $r \doteq 0.97$.

If fed in the mode matching algorithm, convective modes trigger numerical instability of the algorithm, which in turn, results in the dramatic loss of accuracy in the regions of clustering. An alternative is, for example, to use only a limited number of hydrodynamic modes in the mode matching procedure chosen in such a way as to insure that the value of the norm of the difference between the approximated function and the approximation, say, in the central control plane $x=0.34 \mathrm{~m}$

$$
\varepsilon_{N}=\frac{1}{N} \sum_{i=0}^{N-1}\left|\mathcal{P}_{1}\left(r_{i}\right)-\sum_{\mu \in \mu_{0} \cup \mu_{+} \cup \mu_{-}} A_{\mu} P_{\mu}\left(r_{i}\right) \exp \left(i k_{\mu} x_{1}\right)\right|
$$

is the lowest. Here $r=r_{i}, i=0,1,2 \ldots N-1$ are the nodes of the uniform computational grid used to integrate the modal equation. 


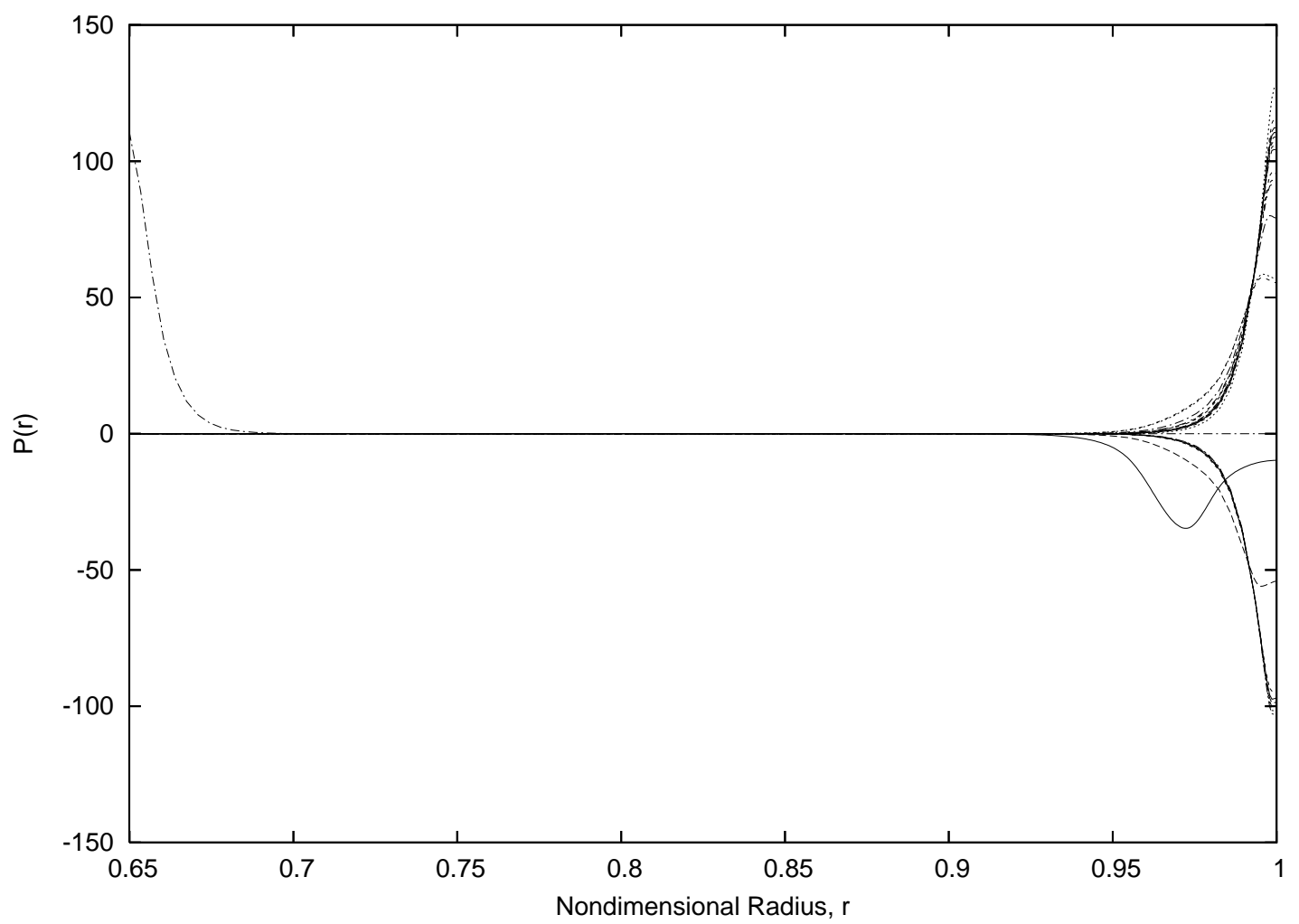

Figure 5. Hydrodynamic modes versus $r$. The modes localised near $r \doteq 1$ are due to the boundary-layer flow near the outer wall. Notice the similarity of their shape and the clustering effect.

This approach shows that if all hydrodynamic modes depicted in figure 5 are used in the approximation procedure, the approximation error $\varepsilon_{N}$ reaches the highest value equal to $0.947 \cdot 10^{-4}$. However this error can be decreased to only $0.14 \cdot 10^{-4}$ by removal of any two of the three clustering modes near the value of $k_{\mu} \doteq 122$ in figure 4 . Further dramatic decrease in the approximation error can be achieved by removal of all hydrodynamic modes with the exception of the two modes which are the closest to the walls of the duct, the mode generated by the inflection point at $r \doteq 0.97$ and the mode closest to the other inflection point $r \doteq 0.984$. In this case $\varepsilon_{N}=0.0395 \cdot 10^{-4}$. Subsequent removal of the mode corresponding to the inflection point $r \doteq 0.984$ which lies well in the outer wall boundary layer results in a slight further improvement in accuracy, with $\varepsilon_{N}=0.0393 \cdot 10^{-4}$. This value is the absolute minimum of the approximation error for the chosen set of modes. If the remaining inflection point mode which lies near the outer boundary of the boundary-layer flow (shown by the thick line in figure 5) is also removed, the error grows to $\varepsilon_{N}=0.0413 \cdot 10^{-4}$. If all hydrodynamic modes are removed and only acoustic spectrum is used $\varepsilon_{N}=0.0469 \cdot 10^{-4 \%}$.

The undertaken analysis of the approximation error suggests that in practice it may be sufficient to retain only two hydrodynamic modes which are the closest to the walls and the modes induced by the inflection points of the mean flow profile. Interestingly, it is only the inflection point that lies sufficiently far from the wall that needs to be taken into account. We hasten to note, though, that these results have been obtained for a sheared flow with no swirl. In general, swirling flows are known to generate continuous hydrodynamic spectrum which is of a different physical nature than the spectrum we consider here. Hence, the present findings may not be directly applicable to swirling flows.

The approximation errors for the linear and uniform velocity profiles shown in figure 2 are $\varepsilon_{N}^{l i n}=0.0369$. $10^{-4}$ and $\varepsilon_{N}^{u n i}=0.0468 \cdot 10^{-4}$, respectively. The latter error is almost identical to the value of $\varepsilon_{N}=0.0469$. $10^{-4}$ obtained for the purely acoustic approximation based on the boundary-layer velocity profile. Hence, accurate modelling of refraction effects does not seem to be important for the current engine. Comparison of $\varepsilon_{N}^{u n i}$ to the best fit error for the boundary-layer mean flow profile $\varepsilon_{N}^{b l}=0.0393 \cdot 10^{-4}$ shows that, as expected, inclusion of the hydrodynamic modes gives perceptible improvement over the purely acoustic uniform flow approximation. However, the fact that $\varepsilon_{N}^{l i n}$ is smaller than $\varepsilon_{N}^{b l}$ suggests that technically it is 


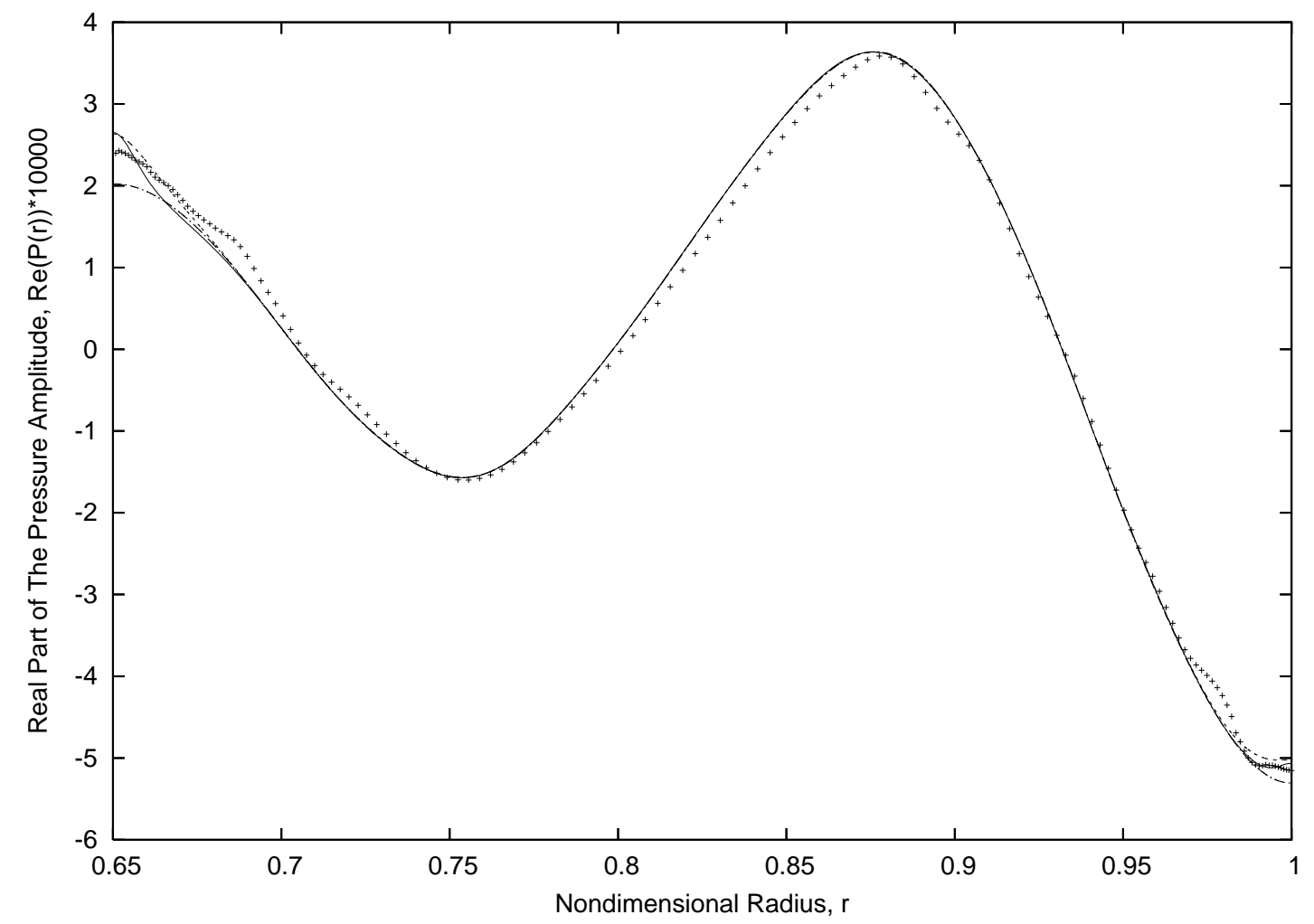

Figure 6. Triple plane pressure matching results for the real part of pressure versus radial coordinate $r$ in the control plane $x=0.34 \mathrm{~m}$ : asterisk - original RR data, dash-doted line - approximation based on the uniform mean flow profile; dashed line - approximation based on the linear mean flow profile; solid line - approximation based on the boundary-layer profile. 
more advantageous to use the two hydrodynamic modes which result from the extrapolation of the core of the sheared mean flow profile into the boundary-layer regions, than the modes found by direct modelling of the boundary-layer profile. Presumably, the reason for this is that in the numerical computation only a finite subset of the continuous spectrum is available, while the majority of the modes are inevitably missed, and to insure proper mode sampling one has to work with a very fine grid to minimize $\varepsilon_{N}^{b l}$. Hence the use of an inviscid, but sheared, profile seems to be an attractive practical option.

Figures 6 and 7 illustrate the quality of pressure approximations for uniform, linear and boundary-layer mean flow profiles corresponding to the above values of $\varepsilon_{N}^{u n i}, \varepsilon_{N}^{l i n}$ and $\varepsilon_{N}^{b l}$, respectively. The values of the pressure amplitude and radial coordinate $r$ are nondimensionalised with $\bar{\rho}(d) \bar{c}^{2}(d)$ and the outer radius $d$, respectively. It can be seen from these figures that all the approximations are rather accurate and give identical results in the main part of the duct, where the mean flow is inviscid. In the boundary-layer regions inclusion of hydrodynamic modes either in the case of the linear profile of the boundary-layer profile improves the quality of the approximation over the purely acoustic case. The use of two hydrodynamic modes near the outer wall - one corresponding to the inflection point $r \doteq 0.97$ and the other corresponding to the mode with the largest available $k_{\mu}$ for the boundary-layer profile - gives a slightly better approximation than the approximation based on the linear profile with only one hydrodynamic mode near the outer wall. However, the differences between the two approximations are very small.

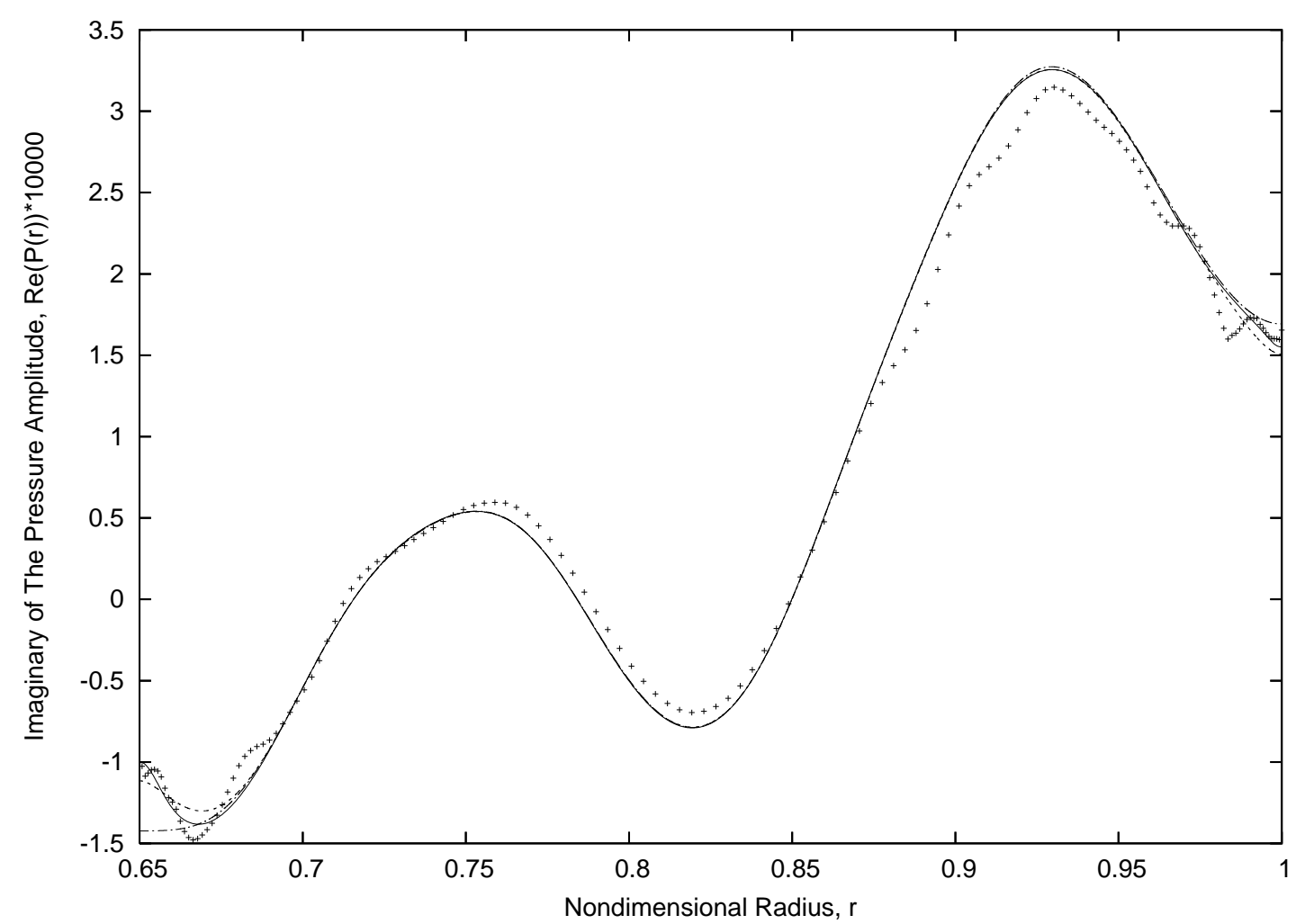

Figure 7. Triple plane pressure matching results for the imaginary part of pressure versus radial coordinate $r$ in the control plane $x=0.34 \mathrm{~m}$ : asterisk - original RR data, dash-doted line - approximation based on the uniform mean flow profile; dashed line - approximation based on the linear mean flow profile; solid line approximation based on the boundary-layer profile.

\section{Conclusions}

The triple plane matching method recently used by Ovenden \& Rienstra (2004) to decouple the problems of noise generation and propagation for turbomachinery applications has been extended here to nonuniform ducted flows.

The modal approximations based on nonuniform slip-stream mean flow profiles give accurate results while inclusion of hydrodynamic modes allows for further improvement over purely acoustic modal expansion near 
the duct walls. Although theoretically possible, modelling of the subtle flow structure in the boundarylayer regions near the duct walls based on modal expansions which include convected modes is found to be computationally rather expensive and not necessarily advantageous. Since the slip-stream approximation is already rather accurate and much easier to obtain numerically, it is suggested that in practical computations the modes associated with mean flow features due to the boundary-layer should be discarded.

\section{Acknowledgements}

The author is grateful to Dr. S.W. Rienstra for helpful comments and discussions during the author's visit to The Netherlands which was supported by the "Messiaen" European collaborative project 'Methods fot the efficient simulation of aircraft engine noise' (EU Technical Officer Dietrich Knörzer and Coordinator Jean-Louis Migeot, Free Field Technologies).

\section{References}

${ }^{1}$ Cooper, A.J., Peake, N., 2005 Upstream-radiated rotor-stator interaction noise in mean swirling flow. J. Fluid Mech., vol. 532, pp. 219-250.

${ }^{2}$ Eversman, W. 1991 Theoretical model for duct acoustic propagation and radiation. In Aeroacoustics of Flight Vehicles: Theory and Practice. Volume 2: Noise Control (Ed. H.H. Hubbard), chapter 13, p. 101 - 163.

${ }^{3}$ Golubev, V.V, Atassi, H.M., 1998 Acoustic vorticity waves in swirling flows. J. of Sound and Vibration, vol. 209(2), pp.203-222.

${ }^{4}$ Nijboer, R. 2001 Eigenvalues and eigenfunctions of ducted swirling flows. AIAA 2001-2178, pp. 1-11.

${ }^{5}$ Ovenden, N.C., Rienstra, S.W. 2004 Mode-matching strategies in slowly varying engine ducts. AIAA Journal Vol. 42, No 9, pp. $1832-1840$.

${ }^{6}$ Tam, C.K.W., Auriault, L., 1998 The wave modes in ducted swirling flows. J. Fluid Mech., vol. 371, pp.1-20. 